renography measured $80 \%$ of isotopic uptake over the normal sized left kidney. Histological examination of a biopsy specimen from this kidney showed pronounced glomerulosclerosis, tubular atrophy and fibrosis, and round cell infiltration of the interstitium, but urate crystals were not identified. Despite good control of hypertension and hyperuricaemia the GFR gradually fell over the next two years when malignant hypertension and a rapid deterioration in renal function supervened and the patient has now been admitted to a programme of renal dialysis.

Metabolic studies were performed on the patient's siblings and her close maternal relatives; the paternal relatives were not available for study. Subjects were questioned for a history of gout and resting blood pressure was measured. Serum concentrations and 24-hour urinary excretion of urate were measured by a uricase method on samples taken from subjects receiving a low purine diet. GFR was determined by EDTA clearance. The family tree is shown.

Of the 20 subjects studied, GFR was reduced in half (mean 52\% of expected, range 15-74\%). Five of these also had gout or hyperuricaemia that was associated with decreased renal clearance of urate. A sixth subject had reduced GFR and urate clearance without hyperuricaemia. These findings were detected by the second decade, common by the third, and most pronounced in elder members of this kindred. Seven subjects with renal impairment were also hypertensive.

The familial occurrence of juvenile
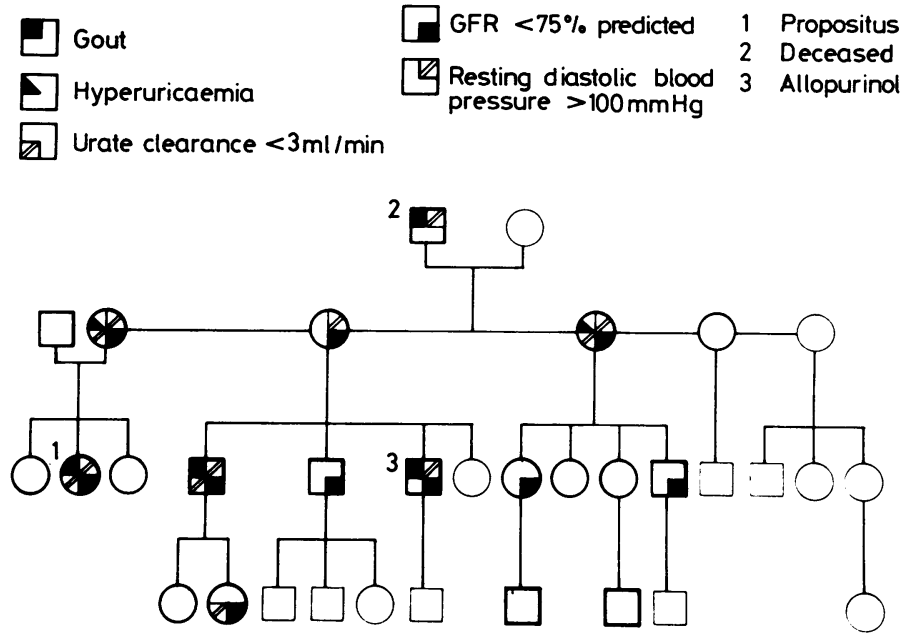

Fig. 1 Family tree. Heavy lines denote subjects studied, circles females, and squares males.

onset of gout associated with rapidly deteriorating renal function has been reviewed. ${ }^{1}$ Inheritance appeared to be dominant and sometimes exclusive in females. The usual pattern was of hyperuricaemia predating or being disproportionate to the degree of renal impairment, though renal failure without hyperuricaemia was occasionally observed in these families. It remains undetermined whether the hyperuricaemia was responsible for, or consequent to, disordered renal function. ${ }^{23}$

As the constant metabolic abnormality in this family was a reduction in the GFR, with impaired urate clearance and hyperuricaemia occurring only in elder members, it appears that a renal abnormality is the primary event rather than hyperuricaemia. In contrast to previous reports, the family history of gout and renal failure was not striking in the case described here and might easily have been overlooked. This familial association may be more common than suggested by the few families described.

\section{References}

1 Simmonds H A, Warren D J, Cameron J S, et al. Familial gout and renal failure in young women. Clin Nephrol 1980; 14: 176-82.

2 Fessel W J. Renal outcomes of gout and hyperuricaemia. Am J Med 1979; 67: 74-82.

3 Rosenfeld J B. Effect of long term allopurinol on serial GFR in normotensive and hypertensive hyperuricaemic subjects. Adv Exp Med Biol 1974; 41B: 581-96.

\title{
Renal failure associated with crystal-induced nephropathy and gout in a baby boy
}

\author{
H.A. SIMMONDS, ${ }^{1} \mathrm{~T} . \mathrm{M}$. BARRATT ${ }^{2} \mathrm{M} . \mathrm{J}$. DILLON, ${ }^{2} \mathrm{P} . \mathrm{C}$. HOLLAND, ${ }^{2}$ \\ J.R. PINCOTT, ${ }^{3}$ L. D. FAIRBANKS, ${ }^{1}$ J. H. STUTCHBURY, AND J.S. CAMERON ${ }^{1}$
}

From the 'Purine Laboratory and Renal Unit, Guy's Hospital Medical School, London, ${ }^{2}$ Renal Unit, Great Ormond Street Hospital for Sick Children, and ${ }^{3}$ Department of Pathology, Great Ormond Street Hospital for Sick Children, London

There has been much controversy over the relative roles of crystal deposition, vascular disease, and age in the genesis of the renal lesion in gout, where nephropathy was formerly common but is now extremely rare. ${ }^{2}$
Considerable debate as to whether the origin of the lesion was interstitial ${ }^{3}$ or stemmed from intratubular deposition 
of uric acid ${ }^{4}$ has also occurred. We have argued for the primacy of intratubular crystal deposition in the past ${ }^{4}$ based on studies using an animal model, as well as patients with inherited disorders resulting in gout and/or nephropathy. ${ }^{5-7}$ We here present data on severe renal damage associated with tubulo-interstitial deposition of uric acid/urate in an infant $^{8}$ with hypoxanthine-guanine phosphoribosyltransferase deficiency (HGPRT:EC 2.3.2.8), in the absence of hypertension or vascular pathology.

CASE HISTORY

A 5 week old boy was the first child of healthy unrelated parents. From 3 weeks he thrived poorly, had feeding difficulties, and was extremely irritable. On admission the thumb and first two fingers of the right hand were red, swollen, and painful. Neurologically, he was slightly hypotonic. Plasma creatinine was 350 $\mu \mathrm{mol} / 1(3.9 \mathrm{mg} / 100 \mathrm{ml})$ and plasma urate disproportionately high at 1.13 $\mathrm{mmol} / \mathrm{l}(9 \mathrm{mg} / 100 \mathrm{ml})$. However, ratio of urine uric acid to creatinine ( $\mathrm{mmol} / \mathrm{mmol}$ ) was $1 \cdot 17: 1$, which is within the normal range for a child of this age. ${ }^{8}$

Plain abdominal $x$-ray film showed no radio-opaque calculi, but renal ultrasound showed both kidneys were bright, suggesting a crystal nephropathy. ${ }^{8} \mathrm{~A}$ renal biopsy showed crystals in both tubules and interstitium in a cryostat section under polarised light. There was much tubular atrophy with extensive tubular epithelial giant cell transformation in the cortex and medulla. All crystals dissolved on fixing in formalin.

HGPRT activity was $<0.01$ $\mathrm{nmol} / \mathrm{mg} \mathrm{Hb} / \mathrm{h}$ in lysed red cells, $<0.9$ $\mathrm{nmol} / \mathrm{mg}$ protein $/ \mathrm{h}$ in fibroblasts and $<0.2 \%$ of normal in intact red cells. ${ }^{58}$ Incorporation of labelled hypoxyanthine into nucleotides by intact fibroblasts was $6 \%$ of control, which is low on the criteria of Page et al. ${ }^{9}$ These results confirm severe HGPRT deficiency. ${ }^{58}$

Treatment has consisted of allopurinol $5-10 \mathrm{mg} / \mathrm{kg} / 24 \mathrm{~h}$ and sodium bicarbonate. Plasma uric acid on discharge had fallen to $0.5 \mathrm{mmol} / 1$ $(8 \mathrm{mg} / 100 \mathrm{ml})$, plasma creatinine to 90 $\mu \mathrm{mol} / \mathrm{l}(1.0 \mathrm{mg} / 100 \mathrm{ml})$. The allopurinol dose has subsequently required careful monitoring.

\section{COMMENT}

The onset of clinical gout at 5 weeks of age must be unique. The association of gout and renal failure due to crystal-induced nephropathy is also rarely seen today. ${ }^{12}$ The histological lesion is generally a non-specific interstitial nephritis considered to be due to age, hypertension, or both. ${ }^{1-3}$ Others have defined two specific nephropathies-acute nephropathy due to intratubular uric acid deposition (essentially reversible) as distinct from the slow insidious interstitial deposition of sodium urate from supersaturated body fluids (essentially irreversible). ${ }^{3}$

The lesion in this young child supports our contention from animal studies, as well as a case of 2 , 8-dihydroxyadenine nephropathy, that the initial insult is intratubular, the natural sequelae of which, through basement membrane rupture, is the migration of crystals into the interstitium with subsequent inflammatory response (Fig. 1b) proceeding to fibrosis and permanent renal damage. ${ }^{4-7}$

This hypothesis is supported by the fact that infants maintain a low plasma uric acid through a high urate clearance. $^{6}$ Gross uric acid overproduction in HGPRT deficiency would result in high urinary urate which would provide the initial insult; the raised plasma uric acid must have been secondary to the subsequent renal damage. Plasma urate concentrations (after the immediate neonatal period) are extremely low; to those accustomed to adult values, the raised concentrations in children may thus not appear abnormal. In this child the concentrations were disproportionately high for the degree of renal damage, even for an adult. ${ }^{7} 10$ This provided the only clue to the possibility of uric acid overproduction in this infant, since the usual hallmark, high uric acid excretion on a creatinine basis, was obscured because of the renal damage..$^{5810}$

Adequate control of uric acid concentrations by allopurinol has also presented a problem because of the renal damage, particularly in such a young child. Too high a dose has resulted in retention of oxipurinol, which could potentiate bone marrow depression, ${ }^{10}$ it has also resulted in the excretion of excessive amounts of xanthine, which is more insoluble than uric acid.

This case is important for several reasons. Firstly, it indicates the problems of diagnosis and treatment in HGPRT deficiency when renal function is impaired. ${ }^{510}$ The presence of crystals within the tubules as well as the interstitium in crystotat section only-but not formalin-fixed tissue-underlines the difficulty of identification of uric acid-induced crystal nephropathy histologically, unless special precautions are taken. ${ }^{1}$ The histological picture indicates that crystals can reach the interstitium in the 'gouty kidney', after disintegration of the tubules.

These studies have been supported by grants from the MRC, ARC, Wellcome Trust, and National Fund for Research into Crippling Diseases.

\section{References}

1 Wyngaarden J B, Kelley W N. Gout. In: Stanbury J, B, Wyngaarden J B, Frederickson D S, Goldstein J L, Brown M S, eds. The metabolic basis of inherited disease. 5th ed. New York: McGraw Hill, 1983: 1053-6.

2 Berger L, Yü T F. Renal function in gout IV. An analysis of 524 gouty subjects including long-term follow-up studies. Am J Med 1975; 59: 605-13.

3 Emmerson $B$ T, Row P G. An evaluation of the pathogenesis of the gouty kidney. Kidney Int 1975; 8: 65-71.

4 Farebrother D A, Pincott J R, Simmonds H A, Warren D J, Dillon M J, Cameron J S. Uric acid crystal-induced nephropathy: evidence for a specific renal lesion in a gouty family. $J$ Pathol 1981; 125: 159-68.

5 Dillon M J, Simmonds H A, Barratt T M, Fairbanks L D, Holland P C. Problems in diagnosis and treatment of adenine and hypoxanthine-guanine phosphoribosyltransferase deficiency. $J$ Clin Chem Clin Biochem 1982; 20: 364.

6 Simmonds H A, Warren D J, Cameron J S, Potter C F, Farebrother D A. Familial gout and renal failure in young women. Clin Nephrol 1980; 14: 176-82.

7 Cameron J S, Simmonds H A. Uric acid gout and the kidney.J Clin Pathol 1981; 34: 1245-54.

8 Holland P C, Dillon M J, Pincott J, Simmonds H A, Barratt T M. Hypo$x$ anthine guanine phosphoribosyltransferase deficiency presenting as renal failure in infancy. (in press). 
9 Page T, Bakay B, Nissinen E, Nyhan W L. Hypoxanthine-guanine phosphoribosyltransferase variants: correlations of clinical phenotype with enzyme activity. Journal of Inherited Metabolic Diseases 1981; 4: 203-7.
10 Cameron J S, Simmonds H A, Webster D R, Wass V, Sahota A. Problems of diagnosis and in vitro enzyme instability in an adolescent with hypoxanthineguanine phosphoribosyltransferase deficiency presenting in acute renal fail- ure. J Clin Chem Clin Biochem 1982; 20: 353 .

\title{
Lean, dry gout patients
}

\author{
L. GAIL DARLINGTON \\ From the Epsom District Hospital, Epsom, Surrey
}

Hypertriglyceridaemia is common in gout. ${ }^{2}$ Obesity may increase triglyceride concentrations, as may alcohol, ${ }^{3}$ but it remains uncertain whether obesity and alcohol, alone or in combination, are sufficient to explain the hyperlipidaemia in all cases. We looked for abnormal lipid concentrations in non-obese patients with gout who drank little or no alcohol to determine whether the hy perprebetalipoprote in ae $\mathrm{mia}$ associated with gout occurred in such a lean and abstemious group.

All patients were of desirable weight or less for their age and frame ${ }^{4}$ and no patient drank more than one pint of beer per day or its equivalent. Such patients are rare, and only seven were found in four years from a busy clinic.

Fasting concentrations of lipid and lipoprotein were measured in serum at a laboratory with its own control population.' Serum uric acid concentrations were determined for the patients with gout but unfortunately data for the control population were not available.

Readings for serum cholesterol, triglyceride, $\beta$-lipoprotein and prebeta lipoprotein concentrations in the patients with gout lay within 2 standard deviations of the corresponding mean for controls. This means that they were within the $95 \%$ confidence limits for the control population and it is therefore unlikely that there is any real difference between the patients with gout and control populations.

In spite of the small numbers of these, 'lean, dry' patients, the results revealed no intrinsic hyperlipidaemia in subjects with gout when obesity and an excess of alcohol were removed as causes of hypertriglyceridaemia.

\section{References}

1 Darlington L G, Scott J T. Plasma lipid levels in gout. Ann Rheum Dis 1972; 31: 487-9.

2 Mielants H, Veys E M, de Weerdt A. Gout and its relations to lipid metabolism. I. Serum uric acid, lipid and lipoprotein levels in gout. Ann Rheum Dis 1973; 32: 501-5.

3 Gibson T, Grahame R. Gout and hyperlipidaemia. Ann Rheum Dis 1974; 33: 298-303.

4 Metropolitan Life Insurance Company. Build and blood pressure study. Chicago: Society of Actuaries, Ill. 1959.

5 Slack J, Noble N, Meade T W, North W $R$ S. Lipid and lipoprotein concentrations in 1,604 men and women in working populations in north-west London. Br Med J 1977; ii: 353-6.

\section{Cardiovascular disease and gout: a function of sex and age?}

\author{
D. G. MACFARLANE
}

From the University Department of Medicine, Bristol Royal Infirmary, Bristol

Hypertriglyceridaemia is common in patients with gout and hyperuricaemia but it is still not known whether this results from a link between purine and lipoprotein metabolism or whether they occur together due to other associated facts, particularly obesity and high alcohol intake, both of which are commonly found in patients with hyperuricaemia. ${ }^{1}$ Nor is it firmly established whether patients with gout are, in fact, predisposed to premature cardiovascular disease, and, if so, whether the raised serum uric acid concentration operates as an independent risk factor, or only via its association with hypertriglyceridaemia and hypertension, which are established as cardiovascular risk factors in their own right. ${ }^{2}$ Another possibility-namely, that a raised serum uric acid concentration causes platelet hyperaggregatability and hence thrombosis-has recently been investigated in our unit with negative results. $^{3}$

Over a period of two and half years 